\title{
Floating-Disk Parylene Microvalves for Self-Pressure-Regulating Flow Controls
}

\author{
Po-Jui Chen, Student Member, IEEE, Student Member, ASME, Damien C. Rodger, Member, IEEE, \\ Mark S. Humayun, Member, IEEE, and Yu-Chong Tai, Fellow, IEEE
}

\begin{abstract}
This paper presents the first parylene-based floatingdisk microvalve with self-pressure-regulating characteristics for various microfluidic applications. By incorporating a freefloating disk diaphragm with no anchoring/tethering structures to constrain its movement, the microvalve realizes configurable pressure-based flow-shunting functions in a stand-alone fashion. Its passive operation eliminates the need for power sources or the external actuation of the device. A multilayer polymer surface-micromachining technology is utilized for device fabrication by exploiting parylene $C$ (poly-chloro-p-xylylene) as the biocompatible structural material for high mechanical compliance as compared with other conventional thin-film materials. Experimental results successfully demonstrate that the inchannel microvalves control water flows in the following two different shunt designs: 1) a nearly ideal regular check valve with zero forward-cracking pressure, zero reverse leakage, and $1.25 \times 10^{13}-2.09 \times 10^{13} \mathrm{~N} \cdot \mathrm{s} / \mathrm{m}^{5}(0.03-0.05 \mathrm{psi} \cdot \mathrm{min} / \mu \mathrm{L}, 1.55-$ $2.59 \mathrm{mmHg} \cdot \min / \mu \mathrm{L}$ ) of fluidic resistance; and 2) a pressurebandpass check valve with $0-100 \mathrm{mmHg}$ and $0-10 \mu \mathrm{L} / \mathrm{min}$ of pressure and flow rate regulation ranges, respectively, as well as $4.88 \times 10^{13} \mathrm{~N} \cdot \mathrm{s} / \mathrm{m}^{5}(0.12 \mathrm{psi} \cdot \mathrm{min} / \mu \mathrm{L}, 6.08 \mathrm{mmHg} \cdot \mathrm{min} / \mu \mathrm{L})$ of fluidic resistance in the forward conductive region. Such a biocompatible and implantable microvalve has the great potential of being integrated in microfluidic systems to facilitate effective microflow control for lab-on-a-chip and biomedical applications.

[2008-0055]
\end{abstract}

Index Terms-Flow control, fluidics, mechanical engineering, microelectromechanical devices, micromachining, valves.

\section{INTRODUCTION}

$\mathbf{F}$ LOW CONTROLS enabled by passive microfluidic valves have great a value in minimizing integration and operation

Manuscript received March 3, 2008; revised June 23, 2008. First published October 28, 2008; current version published December 4, 2008. This work was supported in part by the Engineering Research Centers Program of the National Science Foundation under NSF Award EEC-0310723 and in part by Bausch and Lomb. Earlier versions of this paper were presented at the 20th IEEE International Conference on Micro Electro Mechanical Systems, Kobe, Japan, January 21-25, 2007, and the 21st IEEE International Conference on Micro Electro Mechanical Systems, Tucson, AZ, January 13-17, 2008. Subject Editor Y. Zohar.

P.-J. Chen is with the Department of Electrical Engineering, Division of Engineering and Applied Science, California Institute of Technology, Pasadena, CA 91125 USA (e-mail: pjchen@mems.caltech.edu).

D. C. Rodger is with the Keck School of Medicine, University of Southern California, Los Angeles, CA 90033 USA.

M. S. Humayun is with the Doheny Eye Institute, Los Angeles, CA 90033 USA, and also with the Keck School of Medicine, University of Southern California, Los Angeles, CA 90033 USA.

Y.-C. Tai is with the Department of Electrical Engineering and the Department of Bioengineering, Division of Engineering and Applied Science, California Institute of Technology, Pasadena, CA 91125 USA.

Color versions of one or more of the figures in this paper are available online at http://ieeexplore.ieee.org.

Digital Object Identifier 10.1109/JMEMS.2008.2004947

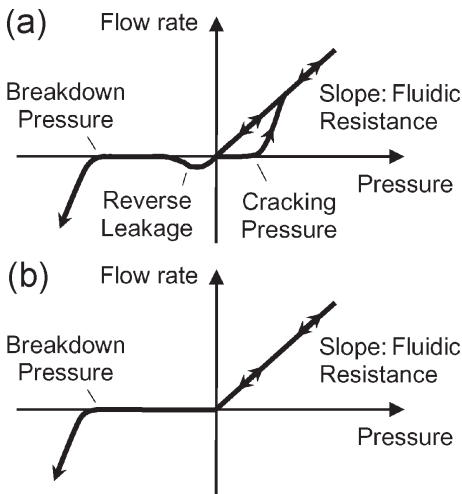

Fig. 1. Fluidic behavior of regular micro check valve. (a) Conventional valve with unwanted forward-cracking pressure and reverse leakage. (b) Valve developed in this paper with nearly ideal flow-shunting curve.

complexity, as well as in manipulating fluids at the microscale in integrated microfluidic systems [1]-[4]. Compared with actively powered valves, passive ones are advantageous in many applications in which design complexity, actuation implementation, power consumption, form factor, and/or integration capability is a major concern. More design parameters need to be included for successful active microvalve development [5]-[7]. In spite of their simplicity, passive microvalves have technical challenges related to their lack of external active components, particularly the forward-cracking pressure and reverse leakage problems shown in Fig. 1(a) in a conventional micro check valve [8]. Ideally, a check valve should have the following three most crucial flow-shunting characteristics: 1) zero reverse leakage; 2) zero forward-cracking pressure; and 3) zero forward fluidic resistance. Although the last criterion is theoretically impossible in pressure-driven flow operation due to fluid marching across the valve structure, the first two could be achieved while they have never been both accomplished at the same time in a single microvalve. Instead, many kinds of micro check valves have been developed for resolving a single issue, and they are recognized as "normally closed" or "normally open" valves with their respective advantages and disadvantages. As a result, these two imperfections limit the practical use of microvalves for flow control, specifically in miniature pressure/flow rate operations, thus leaving room for microvalve improvement. This paper, therefore, first aims to develop a micro check valve with greatly refined valve performance without the aforementioned drawbacks. By using a floating-disk design, the proposed microvalve is the first micro check valve known, to date, which eliminates both reverse leakage and forward-cracking pressure as a nearly ideal regular 


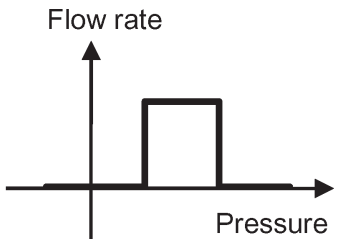

(a)

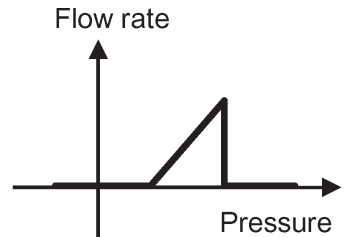

(b)
Fig. 2. Fluidic behavior of pressure-bandpass micro check valve. (a) Ideal valve. (b) Valve developed in this paper with limited pressure/flow rate rising slope due to the fluidic resistance in practice.

check valve [9] with the pressure/flow rate profile shown in Fig. 1(b). It provides a more suitable solution to chip-based valved flow controls with more efficient fluid manipulation.

Other than using such a floating-disk valve as a regular check valve, it can be extensively modified to be a unique unpowered pressure-bandpass check valve [10], with the flow control profile as shown in Fig. 2. Different from a regular check valve that only switches flows on/off with positive or negative pressure loading under forward or backward injection, the pressure-bandpass check valve further regulates the flow within a pressure-loading range. This regulation profile, with highlighted low-off and high-off threshold characteristics, substantially favors the modified floating-disk valve for certain implantable medical microdevices such as drug-delivery [11] and biofluid-transport [8], [12]-[14] devices in which hypotonic or excessive pressures must be prevented with safety considerations that the associated physiological conditions are highly sensitive to the pressure parameter. For example, in physical intraocular pressure (IOP) regulation for glaucoma patients, draining eye fluid within a customized pressure band protects the eye both from hypotony when the IOP is lower than the normal value (e.g., $10 \mathrm{mmHg}$ ) and from excessive high-pressure high-flow-rate drainage when the IOP acutely spikes (e.g., 50-80 mmHg) by routine external interferences such as rubbing or pressing on the eye. These features can be adapted in all biomedical flow controls for safe and effective regulation within a range of interest. By having the exclusive self-pressure-regulating behavior, the modified floatingdisk microvalve serves a stand-alone alternative for greatly reducing design and processing constraints while achieving identical functionality when compared with the previously reported dual-valved paradigm [8]. Using parylene $\mathrm{C}$ here as the main structural material facilitates not only the high mechanical compliance of the floating disk diaphragm but also the high biocompatibility of the proposed polymeric microvalve for being suitable in human health-care and lifescience research.

\section{DESIGN}

\section{A. Regular Micro Check Valve}

The design of the (Design A) nearly ideal regular micro check valve with improved on/off flow control is described first. In order to eliminate both forward-cracking pressure and reverse leakage, the flow-shunting mechanism of a micro check valve requires a moving part which does not have resistance, i.e., does not restore mechanical energy, to any applied

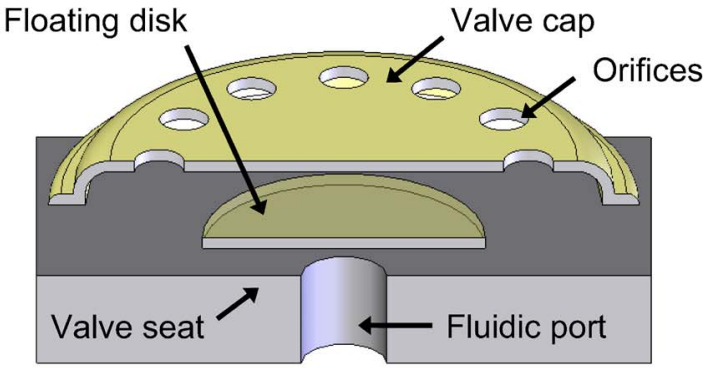

(a)
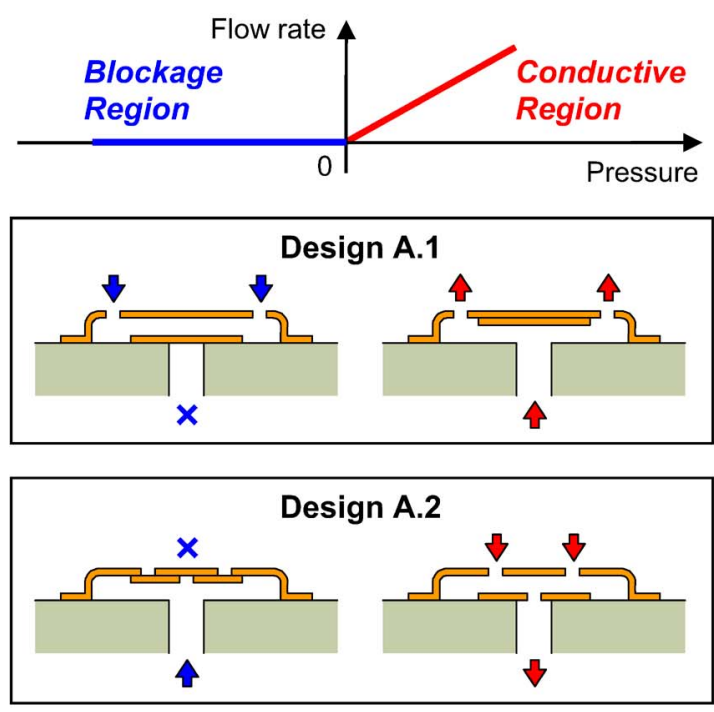

(b)

Fig. 3. (Design A, not to scale) Floating-disk microvalve in regular checkvalve design. (a) Conceptual schematic (cross-sectional exploded view). (b) Flow-shunting curve from the valve with two geometrical design variations which (red arrows on the right side) conduct and (blue arrows on the left side) block flows under different directions of flow injection.

hydraulic pressure. Accordingly, an unclamped floating disk as the essential moving element, shown in Fig. 3(a), is employed with an actuation principle analogous to the free plate of the SiSonic microelectromechanical system (MEMS) microphone (Knowles Electronics, LLC, Itasca, IL) [15]. Being not connected to any anchoring or tethering structure, the disk diaphragm acts as a free plate and moves out of plane immediately under minimal force or pressure loadings. A top cap and a bottom seat are introduced to form a confined space in which the disk diaphragm floats and alters its position in contact with the top cap or bottom seat, depending on the loading direction. Consequently, if the valve disk/seat/cap structures have appropriate orifice locations, the associated fluidic pathway can be shunted on/off by switching the position of the disk diaphragm. This valve configuration enables flow shunting with minimal resistance and hysteresis, which eventually solves the forward-cracking pressure and reverse leakage problems. Two design variations in orifice placements, as shown in Fig. 3(b) (Design A.1: solid disk and valve cap with outer peripheral orifices; Design A.2: disk with central orifice and valve cap with inner peripheral orifices), were implemented to realize the check-valve behaviors with different directions of flow injection. Tolerance of in-plane disk diaphragm movement needs to be addressed to 


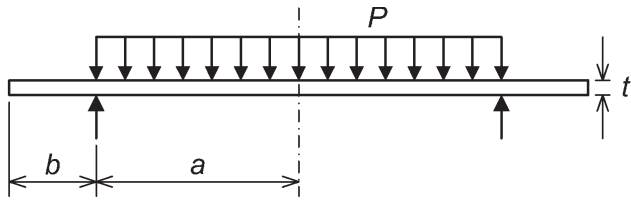

(a)

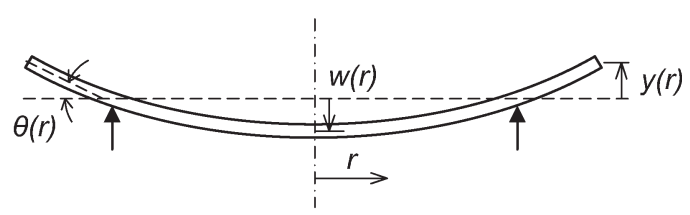

(b)

Fig. 4. Circular plate deformation model in simply supported boundary condition with disk overhang. (a) Loading conditions. (b) Deformation profile.

avoid accidental overlap of the orifices, a potential failure mode of valve operation in practice. Parylene C (poly-chlorop-xylylene) was selected as the structural material because of its ease of processing, CMOS/MEMS processes compatibility, chemical inertness, and biocompatibility (United States Pharmacopeia Class VI grade and ISO 10993 certified) [16], [17], all favorable for the fabrication of the flexible floating disk and the conformation of the microvalve to medical and biological requirements. Its low residual stress varying with processing temperature [18], along with its room-temperature chemical vapor deposition, enables a flat disk diaphragm to be fabricated for secure sealing during valve operation. Such microvalve is designed having an appropriate size (maximum dimension $<500 \mu \mathrm{m}$ ) to demonstrate its feasibility of generic chip-based and microfluidic flow controls. Given by its thin-film nature and corresponding small mass (on the order of $10^{-13} \mathrm{~g}$ ) at the desired device dimensions, the inertial effect of the parylene disk is negligible compared with pressure loadings from the microflows of general interest $(>1 \mathrm{kPa})$ and thus does not have influence on the nearly ideal flow-shunting behavior.

\section{B. Pressure-Bandpass Micro Check Valve}

The nearly ideal regular micro check valve can be modified to have the (Design B) pressure-bandpass flow-shunting behavior by slightly adjusting the boundary condition of the disk diaphragm from the valve seat. As shown in Fig. 4, a disk, in a simply supported condition, when pressed down against a surface (valve seat in this case) under a specified loading area is bendable; with that, the central region of the disk diaphragm bends down while the peripheral region bends oppositely up. Assuming small diaphragm deflection, the diaphragm deflection can be represented as [19], [20]

$w(r)=\frac{P a^{4}}{64 D}\left[1-\left(\frac{r}{a}\right)^{2}\right]\left[\frac{5+\nu}{1+\nu}-\left(\frac{r}{a}\right)^{2}\right], \quad$ for $0<r \leq a$

the angular deformation of the diaphragm in the pressurized area as

$\theta(r) \approx-\frac{d w}{d r}=\frac{P a^{4}}{16 D}\left[\left(\frac{3+\nu}{1+\nu}\right) \frac{r}{a^{2}}-\frac{r^{3}}{a^{4}}\right], \quad$ for $0<r \leq a$

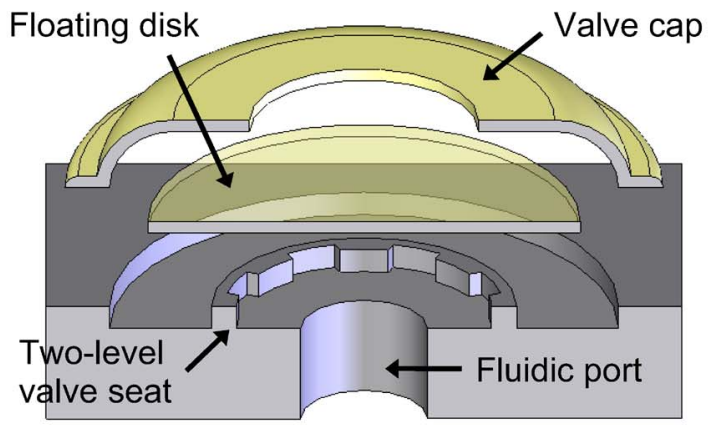

(a)
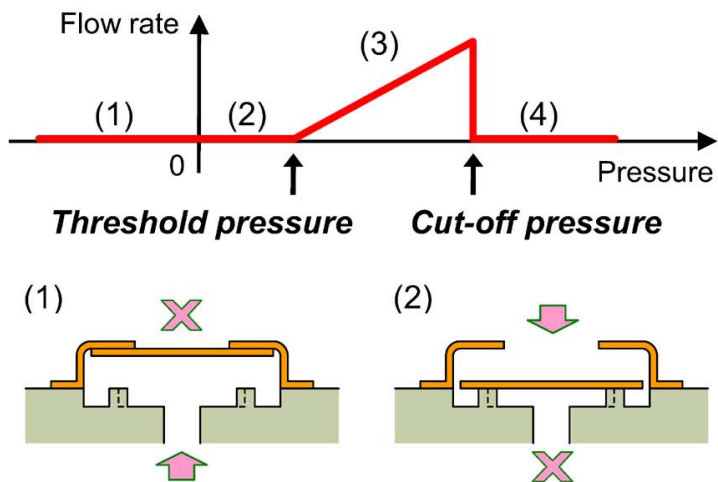

(2)
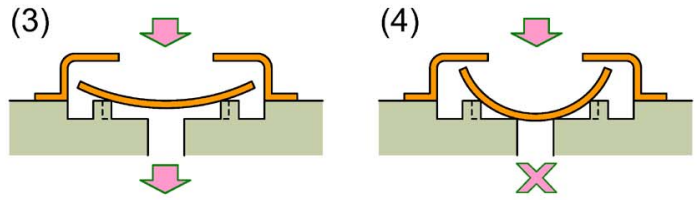

(b)

Fig. 5. (Design B, not to scale) Floating-disk microvalve in pressurebandpass check-valve design. (Top) Conceptual schematic (cross-sectional exploded view). (Bottom) Self-regulation mechanism. Arrows denote flow directions through the valve. Note the relative position between disk diaphragm and radiating trench channels of two-level valve seat in device operation.

and the resulting overhang deflection as

$$
y(r)=(r-a) \tan \theta \approx \frac{P a^{3}(r-a)}{8 D(1+\nu)}, \quad \text { for } r>a
$$

where $a$ is the diaphragm radius applied by a uniformly distributed pressure load $P ; b$ is the diaphragm overhang length; $D=E t^{3} /\left[12\left(1-\nu^{2}\right)\right]$ the flexural rigidity of diaphragm in which $E$ is Young's modulus and $\nu$ is Poisson's ratio of the material; and $t$ is the diaphragm thickness. These equations give the maximum diaphragm deflections at the center and edge, with the sloped angle written as

$$
\begin{aligned}
w_{\text {max }} & =w(0)=\frac{P a^{4}}{64 D}\left(\frac{5+\nu}{1+\nu}\right) \\
\theta(a) & \approx \frac{P a^{3}}{8 D(1+\nu)} \\
y_{\max } & =y(a+b) \approx \frac{P a^{3} b}{8 D(1+\nu)} .
\end{aligned}
$$

Consequently, the unpowered pressure-bandpass micro check valve can be realized by implementing the microvalve design, as shown in Fig. 5, with the fundamental mechanical 
behaviors described by (1)-(6). It comprises a two-level valve seat featuring "leakage-adaptive" radiating fluidic trench channels as the key element. Contact between the disk periphery and the valve seat is removed to overhang the disk outside the supporting rim, leading to no pressure difference across the disk overhang for deliberate disk edge deflection, as shown in Fig. 4. Attributed to the floating disk movement, the fluidic pathway is closed once when the disk is pushed against the valve cap, resulting in the valve being leakage-proof to flows in the backward direction. The forward flows are also blocked when the flexible floating disk is pushed against the valve seat under certain pressure loading and the trench channels of the valve seat remain unexposed. However, if a sufficient pressure is built up to overcome a predetermined threshold point, substantial disk deformation can be created with curved-up overhang so that the beneath-trench channels at the top level of the valve seat are exposed to fluids flowing through them. This behavior defines the threshold pressure of the entire valve operation. On the other hand, the cutoff pressure is defined where the disk is highly bent that it seals the orifice at the bottom level of the valve seat and blocks the flow. These actions jointly lead to the complete pressure-bandpass regulating function of the microvalve. As large deflection $(w \gg t)$ can be expected in such disk deformation, (1)-(6) may need modification in order to accommodate the nonlinear plate-stiffing effect caused by diaphragm stresses, with an example of the corrected load-deflection relationship in the central diaphragm deformation as [20]

$$
\begin{aligned}
& \frac{P a^{4}}{E t^{4}}=C_{1} \frac{w_{\max }}{t}+C_{2}\left(\frac{w_{\max }}{t}\right)^{3} \\
& \frac{\sigma a^{2}}{E t^{2}}=C_{3} \frac{w_{\max }}{t}+C_{4}\left(\frac{w_{\max }}{t}\right)^{2}
\end{aligned}
$$

where $C_{1}=1.016 /(1-\nu), C_{2}=0.376, C_{3}=1.238 /(1-\nu)$, and $C_{4}=0.294$ are the geometry-dependent coefficients and $\sigma$ is the internal stress of the diaphragm. This effect implies that higher pressure loading is required to obtain the same disk deformation estimated in the thin-plate model under the small deflection assumption, and thus needs to be considered for predictable device response. The design points were set to be $15 \mathrm{mmHg}$ in threshold pressure and $60 \mathrm{mmHg}$ in cutoff pressure to demonstrate the feasibility of representative physical IOP regulation using the valved paradigm. These targets were achieved based on high mechanical flexibility (Young's modulus $\sim 4 \mathrm{GPa}$ ) of parylene $\mathrm{C}$ and thin-film structures of the modified microvalve which would be capable of controlling the miniature pressure/flow rate of fluid flows. Such microvalve can be designed having an appropriate size (maximum dimension $<500 \mu \mathrm{m}$ ) to be potentially integrated to a device implant in a form factor suitable for sutureless minimally invasive device implantation [21], [22] toward the aforementioned medical applications.

\section{FABRICATION}

The fabrication processes of the floating-disk microvalves in Designs A and B, as shown in Fig. 6, started with thermally growing 2- $\mu \mathrm{m}$ wet oxide on a double-side-polished silicon wafer. Silicon substrate was exploited here for demonstration of the device concept and fabrication compatibility, while in the implementation perspective, the design and material of the substrate, i.e., the valve seat, could be modified depending on specific application requirements. After patterning the backside oxide using buffered hydrofluoric acid (BHF, Transene Company Inc., Danvers, MA) and photoresist as a mask, deepreactive-ion etching (DRIE) in a PlasmaTherm SLR system (Unaxis Inc., St. Petersburg, FL) was performed to etch the exposed silicon on the backside of the wafer until leaving an approximately $50-\mu \mathrm{m}$-thick membrane. This step was included for defining the fluidic coupling port on the silicon valve seat. Ultimately, these ports were to be etched through so as to provide fluidic access at the inlet and outlet of the microchannel. On the frontside of the wafer, a multilayer polymer surface-micromachining technology was employed to create the valve structures by using parylene C (Specialty Coating Systems Inc., Indianapolis, IN) as the structural layers and the photoresist AZ1518/AZ4620 (Clariant Corporation, Charlotte, $\mathrm{NC}$ ) as the sacrificial layers throughout the entire frontside process. The sacrificial photoresist was hard-baked, at $120{ }^{\circ} \mathrm{C}$ if applicable, for edge-smoothening and degassing purposes. For the microvalves in Design B, another DRIE was performed before polymer micromachining to realize the two-level silicon valve seat. Three parylene $C$ structural layers were deposited and patterned to form the disk diaphragm, the valve cap, and the fluidic channel. The microchannel served the purpose of encapsulating the microvalves as well as connecting the fluidic ports for straightforward forward and backward flow injections in testing afterward. The thin-film structures of the microvalve in Design B had slightly different thicknesses (disk diaphragm: $1 \mu \mathrm{m}$; valve cap: $5 \mu \mathrm{m}$; and fluidic channel: $10 \mu \mathrm{m}$ ) from that in Design A (disk diaphragm: $1.5 \mu \mathrm{m}$; valve cap: $3 \mu \mathrm{m}$; and fluidic channel: $5 \mu \mathrm{m}$ ) for more mechanical robustness. The microchannels had a rectangular inner cross section with $350-\mu \mathrm{m}$ width (500 $\mu \mathrm{m}$ at the valved section), $1800-\mu \mathrm{m}$ length, and different heights regarding different valve designs (Design A: $20 \mu \mathrm{m}$ and Design B: $10 \mu \mathrm{m}$ ) to accommodate the expected higher fluidic resistance from the microvalve in Design B for the reason of convenient pressure/flow rate measurements. Parylene deposition was conducted in a Cookson Electronics PDS 2010 system (Specialty Coating Systems Inc., Indianapolis, IN), and parylene patterning was performed using oxygen plasma etching with photoresist as the mask material. All deposited parylene layers were roughened using a short duration of oxygen plasma treatment to reduce the stiction effect during device operation. To enhance the pressure-loading capacity of the device, gas-phase xenon difluoride $\left(\mathrm{XeF}_{2}\right.$, Nesca Corporation, Pretoria, South Africa) silicon roughening was performed before the second and third parylene layer coatings to physically promote the adhesion of the parylene-silicon interface [23] at the valve cap and the fluidic channel connected to the device substrate. After finishing the frontside process, the remaining silicon membrane on the fluidic port was etched away from the backside of the wafer to create through-wafer holes on the valve seat. Not only did these through-holes enable fluid access to the in-channel microvalves for device operation 
(a)

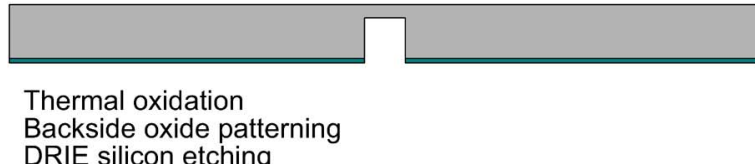

DRIE silicon etching

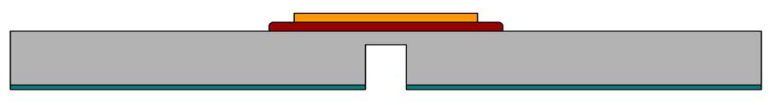

$1^{\text {st }}$ sacrificial photoresist coating and patterning

$1^{\text {st }}$ parylene deposition and patterning (valve disk)

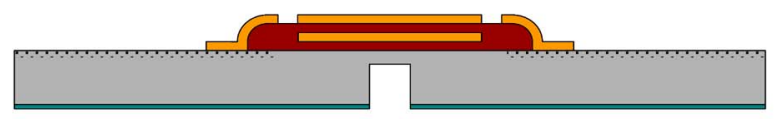

$\mathrm{XeF}_{2}$ silicon roughening

$2^{\text {nd }}$ sacrificial photoresist coating and patterning

$2^{\text {nd }}$ parylene deposition and patterning (valve cap)

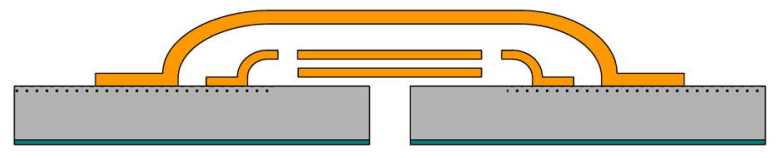

$3^{\text {rd }}$ sacrificial photoresist coating and patterning

$3^{\text {rd }}$ parylene deposition and patterning (microchannel)

Silicon membrane etching

Photoresist stripping (b)
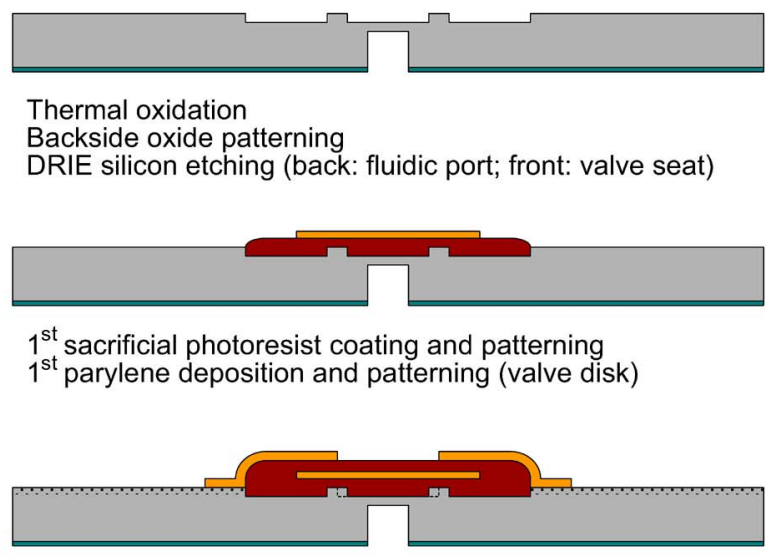

$2^{\text {nd }}$ sacrificial photoresist coating and patterning $\mathrm{XeF}_{2}$ silicon roughening

$2^{\text {nd }}$ parylene deposition and patterning (valve cap)

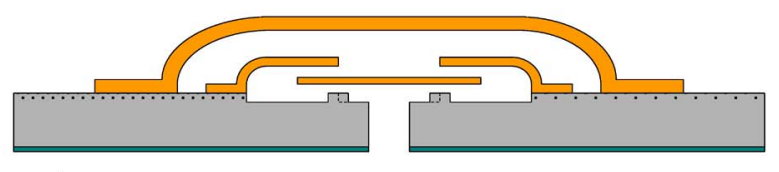

$3^{\text {rd }}$ sacrificial photoresist coating and patterning

$3^{\text {rd }}$ parylene deposition and patterning (microchannel)

Silicon membrane etching

Photoresist stripping

Silicon $\square \mathrm{SiO}_{2} \quad \square$ Photoresist $\square$ Parylene

Fig. 6. Fabrication process flow of floating-disk microvalve. (a) Design A. (b) Design B.

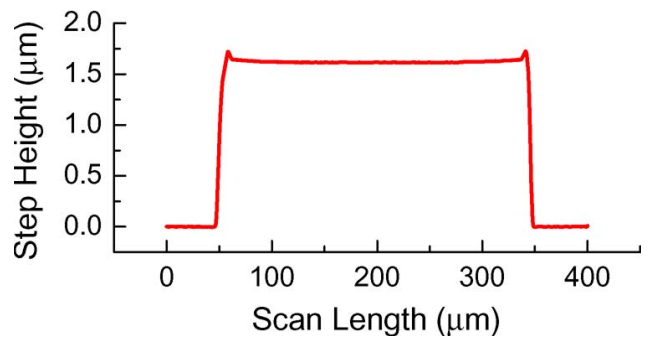

Fig. 7. Profile scan of the actual sacrificial photoresist base for floating disk diaphragm deposition/patterning of microvalves in Design A.

but they also exposed the photoresist so it could be stripped with acetone in the final device release.

Although using photoresist as a sacrificial material is popular in polymer surface-micromachining processes, it raises some challenges to the floating-disk valve fabrication. In principle, the fabricated free-plate disk diaphragm needs to be highly flat for reliable valve behaviors; however, the construction of a flat sacrificial photoresist base for the following disk layer coating/patterning is not trivial, given the involved spin-coating process. For microvalves in Design A, a thin $(\sim 1.5 \mu \mathrm{m})$ layer of photoresist was coated and hard-baked to flatten the sacrificial base as much as possible. A profile scan of the photoresist base, as shown in Fig. 7, confirms that a sufficiently flat surface was obtained at the central region. The peripheral region of the base, however, became rounded and bumpy ( $\sim 7 \%$ higher than the averaged height in the central region shown in Fig. 7) because of photoresist reflow during hard baking. In consequence, the valve disk diaphragm must be defined with a smaller diameter than that of the sacrificial photoresist base to maintain the disk flatness at the edge, which adversely generates planar clearance of the valve disk-seat-cap combination and induces in-plane disk movement in the confines of the valve seat and cap. This concern needs to be taken care of by appropriate geometrical designs of the microvalve in order not to affect its flow-shunting performance. Due to the unavoidable steps on the two-level silicon seat structures for microvalves in Design B, the planarization of the first layer sacrificial photoresist coating required more careful calibration to guarantee the necessary flatness of the free-plate disk diaphragm.

Considering that the valve disk support is crucial for microvalves in Design B to operate, two design variations of the two-level silicon valve seat contours, as shown in Fig. 8, were implemented to study their influence on the following: 1) boundary conditions on the floating disk and 2) overall fluidic conductance. With different numbers and dimensions of the radiating trench channels, the valve seats can have different supporting points for the flexible parylene disk when they are in contact, resulting in different equivalent pressurized areas of the disk and corresponding disk overhang. Therefore, the boundary condition of the microvalve can be tuned to have different disk deformations and different resultant threshold/cutoff pressures. Theoretically, the overall fluidic resistance generated from the valve seat trench channels is also slightly different in the two different contours under the same pressure loading because the disk is deformed to a different extent, which induces different 


\section{Design B.1}
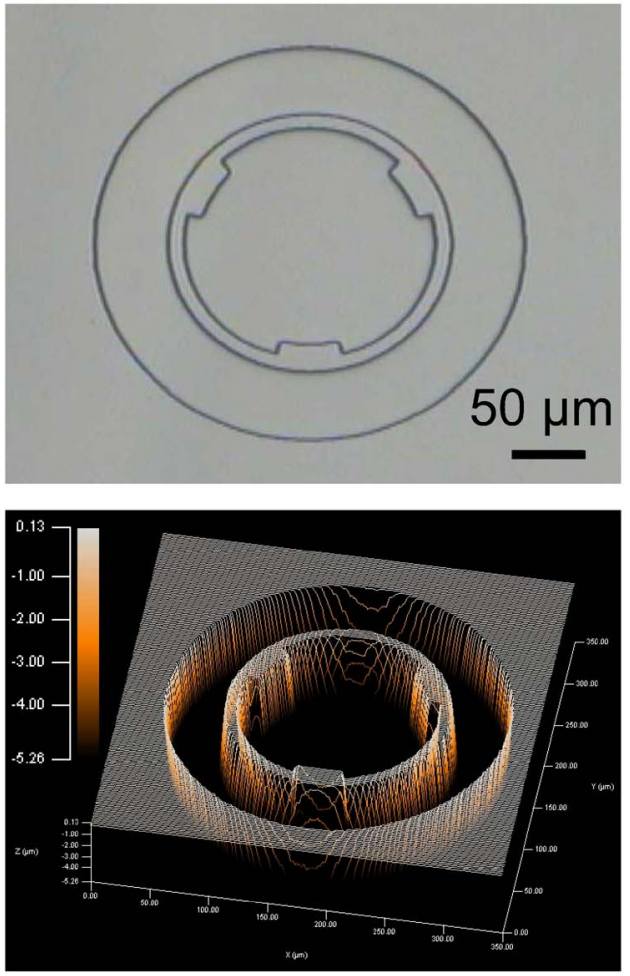

Design B.2
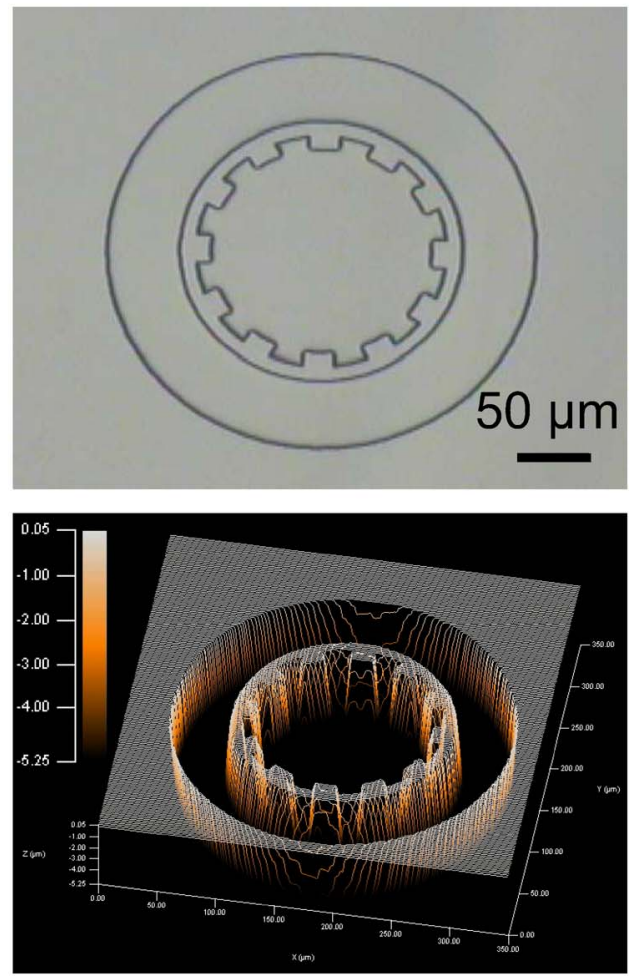

Fig. 8. Micrographs and 3-D surface profile scans of two-level silicon valve seat with two geometrical variations in Design B.

exposures of the valve seat channels for fluidic pathways. Nevertheless, this fluidic resistance change is believed to be insignificant, particularly in miniature flow regulation regimes. Three-dimensional profile scans of the valve seats taken with a P-15 surface profilometer (KLA Tencor Corporation, San Jose, CA) show that the extrusion height was controlled to be $5 \mu \mathrm{m}$ for the predetermined cutoff pressure of interest under the designed device dimensions. More importantly, it was also feasible to planarize this step height using photoresist in a reasonable thickness so as to maintain the process window. The rim width of the valve seat extrusion should be fashioned as small as possible for better realization of the simply supported condition to the disk diaphragm.

The fabricated floating-disk microvalves after device release should be always kept in liquid to prevent stiction of the disk diaphragm. A floating disk diaphragm not connected to any anchoring/tethering structures implies that the disk does not have any support to resist its stiction to other surfaces activated by surface tension during drying, which ends with device failure as shown in Fig. 9. Nevertheless, for liquid flow controls as the majority, the floating-disk valves inside the microchannel do not need to be dried after fabrication while retaining device functionality. Stiction can then be avoided by having no occurrence of the air-liquid-solid interface for the microvalves. As a result, the fabricated devices were partially or fully immersed in water after fabrication so that the fluidic access ports of the microchannel were not exposed to air. Another advantage in liquid flow controls is that the lifetime of such microvalves is naturally extended because minimal wear can be induced using liquids in the valve disk-seat-cap combination. Even if gas flow controls or device packaging in liquid-free envi-

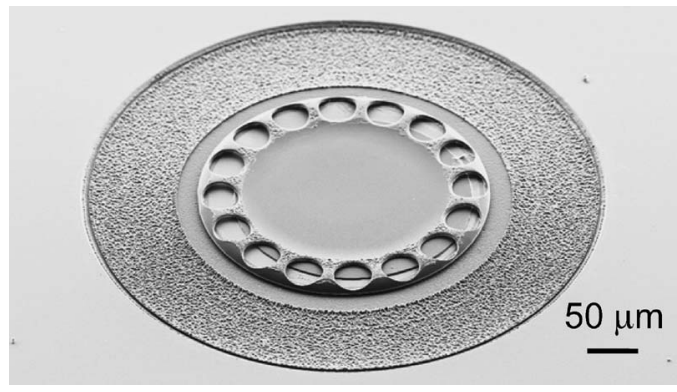

Fig. 9. (Design A.1) SEM image of the fabricated floating-disk microvalve after it is dried in air. The fluidic pathway inside the valve was blocked by the valve disk and cap stuck to the valve seat surface.

ronment is required for certain applications, the microvalves can be rejuvenated by performing a pneumatic disk-popping process after drying [24]. Other antistiction dry device release techniques involving sputtered silicon as a sacrificial material [25], [26] can also be adapted as the alternative. These methods would be valuable for further investigations. Fig. 10 shows the submerged microfabricated in-channel floating-disk parylene microvalves in different designs for different applications. The disk diaphragms were displaced from the center of the valves, as expected, due to their in-plane movement.

\section{EXPERIMENTAL SETUP}

The microfabricated in-channel microvalves were tested using a customized fluidic testing setup as shown in Fig. 11 [8]-[10]. World-to-chip interfacing was accomplished by packaging the diced valved chip in a polyether-ether-ketone jig connected with regular Teflon capillary tubing for liquid transport. 


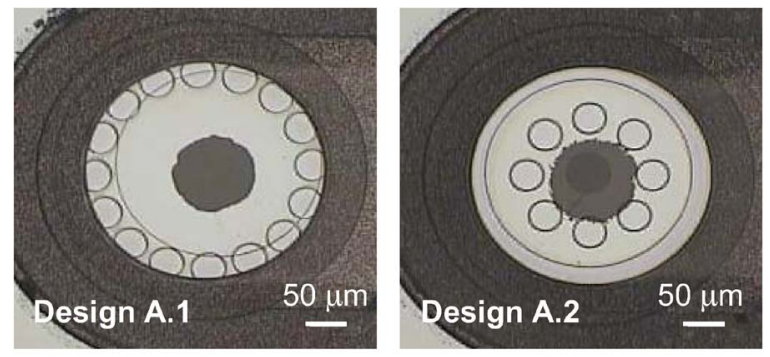

(a)

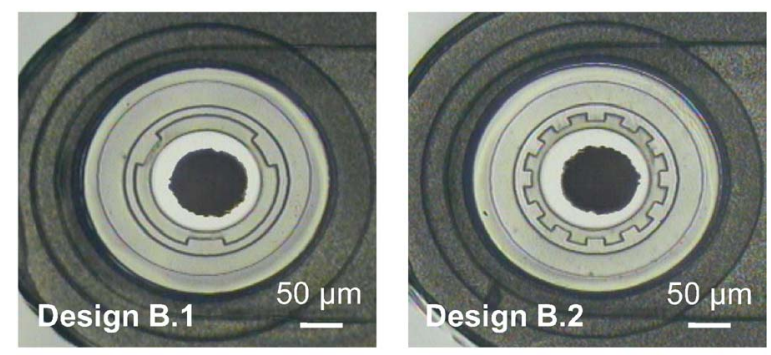

(b)

Fig. 10. (Top view) Micrographs of in-channel floating-disk parylene microvalves immersed in water. (a) (Design A) Regular check valves. (b) (Design B) Pressure-bandpass check valves.

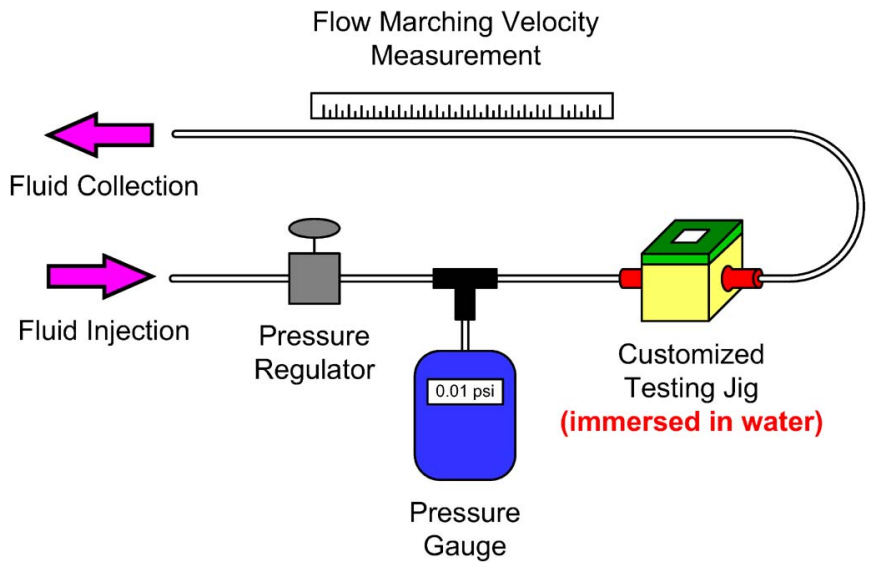

Fig. 11. Schematic of fluidic testing setup for device characterization.

Microflows were introduced to the devices through the jig with access to the backside fluidic ports of the chip. The jig, as well as the packaged device, was immersed in water to avoid an air-liquid-solid interface on the microvalves. Air bubbles in the entire fluidic pathway needed to be completely removed prior to valve characterization to ensure that the microvalves tested in a purely liquid environment. Using water as the working fluid, microflows were injected across the devices using a combination of a commercial off-chip pressure regulator and a pressure gauge with a $0.07 \mathrm{kPa}(\sim 0.01 \mathrm{psi}, \sim 0.5 \mathrm{mmHg})$ tuning resolution. Hydrostatic pressure differences due to any height difference of the column in the testing system were carefully calibrated for accurate pressure control. Flow rate measurement was performed by recording the marching velocity of the water column front in the capillary tube on the back end of the jig before fluid collection. This method was applicable based on the small flow rates (microliter-per-minute level) conducted through the system. In marching velocity recording, given the fact that the resolution was limited to $0.5 \mathrm{~mm}$ in marching dis-

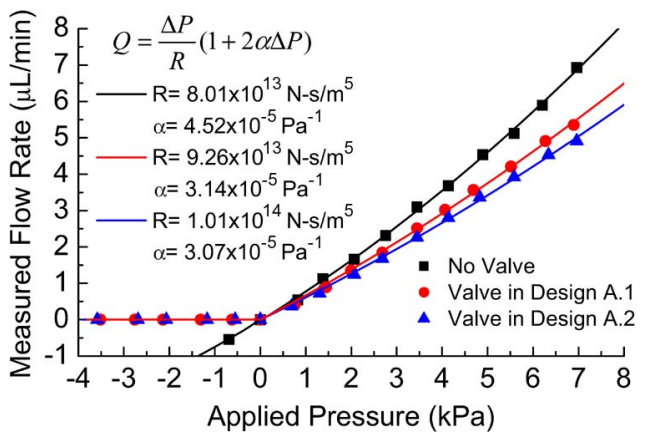

Fig. 12. (Design A) Pressure/flow rate testing results of the in-channel floating-disk regular micro check valves with water as the working fluid. Individual (nonvalved) microchannel was also tested as reference.

tance and $1 \mathrm{~s}$ in marching time in the testing setup, the overall flow rate measurement error was typically $5 \%-15 \%$ depending on the flow rate level. Using these measurement techniques with the stated considerations, the pressure/flow rate behavior of the in-channel microvalves could be well characterized.

\section{RESULTS AND Discussion}

The micromachined in-channel parylene valves were successfully characterized with reliable and repeatable behaviors from the same devices in cyclic operation. All measured pressures shown in the following data are presented in differential gauge format to indicate the additional pressure loading from the injected water relative to atmospheric pressure. Testing results of the floating-disk microvalves in regular and pressurebandpass check-valve designs are, respectively, described in the following two sections.

\section{A. Regular Micro Check Valve}

The functionality of the microvalves in Design A was validated with the pressure/flow rate testing results as shown in Fig. 12. Microchannels with no encapsulated micro check valves were also tested as a reference to compare with the valved channels. It is evident from the plot that no forwardcracking pressure or reverse leakage was observed from the micro check valves in both designs (Designs A.1 and A.2) under pressure loading down to the control limit of the testing setup, which confirms that the floating-disk micro check valves were able to control water flows under minimal applied pressure. On the other hand, the insertion of the microvalve generated additional fluidic resistance to the channel. A structural-fluidic-coupled model with flow-induced channel deformation effect [27], [28] was used to extract the experimental value of fluidic resistance of the devices. For a steady laminar flow inside a microchannel with rectangular inner cross section in a small aspect ratio (height $\ll$ width), the relationship between the applied pressure difference $\Delta P$ and the volumetric flow rate $Q$ can be written as

$$
Q=\frac{\Delta P}{R}(1+2 \alpha \Delta P)
$$


where $R$ is the overall fluidic resistance in the ordinary firstorder pressure-driven flow model and $\alpha$ is the correcting parameter derived from the second-order channel deformation effect. Note that (9) only holds for bulging channels with small deformation, whereas a complete expression must be used for the large deformation case [28]. In order not to increase the modeling and analysis complexity, the pressures under forward flow injection were applied at a level not creating substantial channel deformation to extract the fluidic resistance of the devices so that the data points in Fig. 12 could be accurately fitted using (9). The generated fluidic resistance of the microvalve was $1.25 \times 10^{13} \mathrm{~N} \cdot \mathrm{s} / \mathrm{m}^{5}(0.03 \mathrm{psi} \cdot \min / \mu \mathrm{L})$ in Design A.1 and $2.09 \times 10^{13} \mathrm{~N} \cdot \mathrm{s} / \mathrm{m}^{5}(0.05 \mathrm{psi} \cdot \mathrm{min} / \mu \mathrm{L})$ in Design A.2, $16 \%$ and $26 \%$ more than that from an individual microchannel, respectively. The higher fluidic resistance of the valve in Design A.2 was possibly because the forward flow was conducted downward across the valve at a central area, which leads to more dead volume in the periphery between the disk and cap structures, thus a more resistive fluidic pathway. Under backward flow injection, although the parylene disk diaphragm experienced some plastic deformation after operating under pressure loadings greater than $300 \mathrm{kPa}$, it was still functional in the confines of the valve seat and cap, as the microvalve remained leakage proof. However, device failure to backflows happened at the encapsulating microchannels. Several devices were tested, and the breakdown pressure was found to be in the range of $317-393 \mathrm{kPa}(46-57 \mathrm{psi})$ at which the microchannels could be seriously delaminated from the substrate surface, creating weak points for the sample fluid to leak out from inside the channels. It results from insufficient adhesion at the parylene-silicon interface even after using the silicon-roughening treatment. For an in-depth breakdown pressure test, with a failure mode directly from the floating-disk microvalve instead of the channel, a more robust microchannel with higher pressure-loading capacity, such as the one with parylene-refilled trench anchors [29], should be incorporated in future work. Table I lists several important characteristics of the state-of-the-art surface-micromachined in-channel parylene micro check valves designed for regular on/off flow control for comparison.

\section{B. Pressure-Bandpass Micro Check Valve}

The pressure responses of the microvalves in Design B were first characterized to investigate their threshold and cutoff pressures with different valve seat contours. Testing results summarized in Table II show that their characteristic pressures were within a range suitable for intraocular and most other biomedical applications $(0-100 \mathrm{mmHg})$, in good agreement with expectations based on the design. Although being different with respect to valve seat contours, the microvalves in both Designs B.1 and B.2 obtained similar threshold $(\sim 10$ $20 \mathrm{mmHg})$ and cutoff pressures $(\sim 65-75 \mathrm{mmHg})$, with no significant performance difference using the testing setup. The difference between valves in two design variations, however, is more likely concealed by the variation of pressure testing results caused by imperfect valve disk-seat-cap alignment due to uncontrollable in-plane disk movement during operation. It
TABLE I

COMPARISON OF THE STATE-OF-THE-ART SURFACE-Micromachined IN-ChanNel Parylene Micro CHECK VALVES For REgular ON/OFF Flow CONTROL (WITH WATER AS THE WORKING FLUID)

\begin{tabular}{|c|c|c|}
\hline & Ref. [8] & This work \\
\hline Configuration & Tethered disk & Free-floating disk \\
\hline Occupied area* & $10^{-7} \mathrm{~m}^{2}$ & $10^{-7} \mathrm{~m}^{2}$ \\
\hline Valve void volume & $\begin{array}{l}\sim 3 \mathrm{~nL}\left(\mathrm{NC}^{\dagger}\right) \\
\sim 1 \mathrm{~nL}\left(\mathrm{NO}^{\dagger}\right)\end{array}$ & $\sim 1 \mathrm{~nL}$ \\
\hline Cracking pressure & $\begin{array}{l}\sim 0.2 \mathrm{psi}(\mathrm{NC}) \\
<0.1 \mathrm{psi}(\mathrm{NO})\end{array}$ & $\sim 0$ \\
\hline $\begin{array}{l}\text { Reverse-leakage } \\
\text { stop pressure }\end{array}$ & $\begin{array}{c}\sim 0(\mathrm{NC}) \\
\sim 1.5 \mathrm{psi}(\mathrm{NO})\end{array}$ & $\sim 0$ \\
\hline Breakdown pressure & $>25 \mathrm{psi}$ & $46-57 \mathrm{psi}$ \\
\hline
\end{tabular}

TABLE II

Pressure Testing Results of the Fabricated Microvalves FOR PRESSURE-BANDPASS FlOW CONTROL

\begin{tabular}{ccc}
\hline & Design B.1 & Design B.2 \\
\hline Threshold pressure & $0.31 \pm 0.09 \mathrm{psi}$ & $0.23 \pm 0.04 \mathrm{psi}$ \\
& $(15.9 \pm 4.7 \mathrm{mmHg})$ & $(11.6 \pm 1.9 \mathrm{mmHg})$ \\
& $1.32 \pm 0.06 \mathrm{psi}$ & $1.39 \pm 0.08 \mathrm{psi}$ \\
Cut-off pressure & $(68.6 \pm 3.3 \mathrm{mmHg})$ & $(71.8 \pm 4.2 \mathrm{mmHg})$ \\
\hline
\end{tabular}

Representation format: average \pm standard deviation $(n=5)$.

can change the effective overhang, the pressurized area of the disk diaphragm, and, accordingly, the device performance. It is also known that varied cutoff pressures can be given by the fluidic ports off center from their predetermined location from the double-side pattern alignment process on the wafer, causing more pressure loadings to seal the fluidic port and block the flow. All these factors can change the boundary conditions of the bendable disk as the experimental results had approximately $30 \%-50 \%$ discrepancy from the designed threshold and cutoff pressures. Further study and analysis will need to be conducted to address this issue.

Finally, the pressure/flow rate behavior of the in-channel pressure-bandpass micro check valves was characterized. A sample test result, as shown in Fig. 13, indicated a passively controlled microflow in $0-100 \mathrm{mmHg}$ and $0-10 \mu \mathrm{L} / \mathrm{min}$ of bandpass regulation ranges under the fabricated device dimensions. The microvalves in both designs had similar resultant flow-shunting performance. It is evident from the plot that both low-off and high-off characteristics were successfully achieved in experiments with no observable water flow below the threshold pressure point and greatly reduced flow rate $(<1 \mu \mathrm{L} / \mathrm{min})$ beyond the cutoff pressure point. Regulated flow in the forward conductive region was measured and analyzed having approximately $5.82 \times 10^{13} \mathrm{~N} \cdot \mathrm{s} / \mathrm{m}^{5}$ $(0.14 \mathrm{psi} \cdot \min / \mu \mathrm{L}, 7.25 \mathrm{mmHg} \cdot \min / \mu \mathrm{L})$ of fluidic resistance, comparable to that of valved channels in Design A even with 


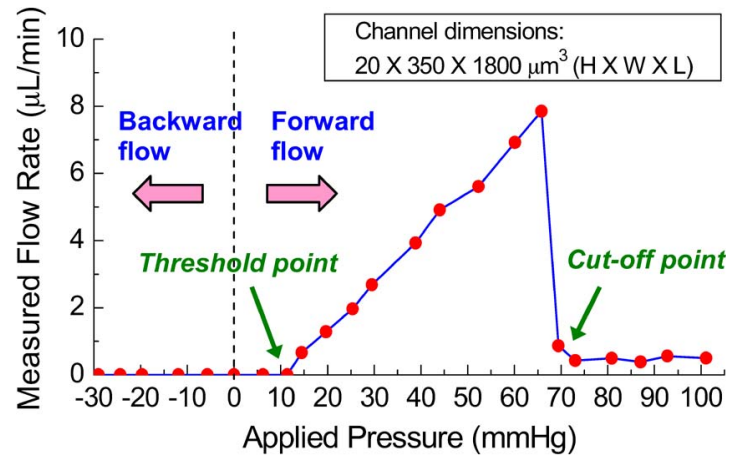

Fig. 13. (Design B.2) Pressure/flow rate testing result of the in-channel floating-disk pressure-bandpass micro check valve showing water flow control in specified regulation profile.

decreased channel fluidic resistance by deliberately increasing the inner height of the microchannel. It is on account of a more resistive fluidic pathway created between the valve disk and the trench channels of the two-level valve seat. The overall fluidic resistance of the microvalves in Design B was calculated to be approximately $4.88 \times 10^{13} \mathrm{~N} \cdot \mathrm{s} / \mathrm{m}^{5}$ $(0.12 \mathrm{psi} \cdot \mathrm{min} / \mu \mathrm{L}, 6.08 \mathrm{mmHg} \cdot \mathrm{min} / \mu \mathrm{L}), 2.3$ and 3.9 times more than that in Designs A.1 and A.2, respectively. As expected, no reverse leakage was observed with pressure loadings higher than $30 \mathrm{psi}(>200 \mathrm{kPa})$ in backward flows. With the successful microvalve demonstration, future work includes fluidic-structural-coupled device analysis and improvement of postfabrication valve disk-seat-cap alignment for device optimization.

\section{CONCLUSION}

A parylene-based floating-disk microvalve has been successfully developed. Its passive operation requires no power consumption or active components to actuate the encapsulated valve disk. A bendable free-plate disk diaphragm not conventionally clamped or tethered facilitates configurable valve functions in a stand-alone fashion. Two different floatingdisk valve designs realize micro check valves with the following two function types: 1) regular on/off flow shunting and 2) pressure-bandpass flow shunting. A multilayer polymer surface-micromachining technology using parylene $\mathrm{C}$ as the biocompatible structural material was utilized to successfully fabricate the in-channel microvalves. Devices tested in a water environment have demonstrated excellent microflow regulations. The microvalves in the regular check-valve design regulate water flows with nearly ideal behaviors, including zero forward-cracking pressure and zero reverse leakage, while additional $1.25 \times 10^{13}-2.09 \times 10^{13} \mathrm{~N} \cdot \mathrm{s} / \mathrm{m}^{5}(0.03-0.05 \mathrm{psi}$. $\min / \mu \mathrm{L}, 1.55-2.59 \mathrm{mmHg} \cdot \min / \mu \mathrm{L}$ ) of fluidic resistance is obtained from the insertion of the valves in the microchannels. The microvalves in the pressure-bandpass check-valve design extensively perform their low-off and high-off characteristics below $\sim 10-20$ - $\mathrm{mmHg}$ and above $\sim 65-75-\mathrm{mmHg}$ pressures, respectively, along with a corresponding $0-10-\mu \mathrm{L} / \mathrm{min}$ flow rate range in the entire flow regulation regime and $4.88 \times$ $10^{13} \mathrm{~N} \cdot \mathrm{s} / \mathrm{m}^{5}(0.12 \mathrm{psi} \cdot \mathrm{min} / \mu \mathrm{L}, 6.08 \mathrm{mmHg} \cdot \min / \mu \mathrm{L})$ of fluidic resistance in the forward conductive region. All microfabricated valves are verified leakage proof to backflows over 30 psi ( $>200 \mathrm{kPa}$ ). Supported by experimental results of the flow-shunting capability and the unique biocompatible property of parylene, the floating-disk microvalve can fulfill self-pressure-regulating biomedical microflow control. Such a device leverages a unique paradigm to facilitate integration in a wide variety of MEMS-based lab-on-a-chip and implantable systems for numerous applications, including, but not limited to, intraocular fluid drainage for physical IOP regulation of glaucoma patients, drug delivery, and biofluid transportation.

\section{ACKNOWLEDGMENT}

The authors would like to thank J. Shih for his valuable comments on fluidic measurements and analysis and T. Roper for his fabrication assistance.

\section{REFERENCES}

[1] A. van den Berg and T. S. J. Lammerink, "Micro total analysis systems: Microfluidic aspects, integration concept and applications," Microsyst. Technol. Chem. Life Sci. Topics Curr. Chem., vol. 194, pp. 21-49, 1998.

[2] S. Shoji, "Fluids for sensor systems," Topics Curr. Chem., vol. 194, pp. 163-188, 1998.

[3] K. W. Oh and C. H. Ahn, "A review of microvalves," J. Micromech. Microeng., vol. 16, no. 5, pp. R13-R39, Mar. 2006.

[4] G. T. A. Kovacs, Micromachined Transducers Sourcebook. Boston, MA: McGraw-Hill, 1998.

[5] S. Sutanto, P. J. Hesketh, and Y. H. Berthelot, "Design, microfabrication and testing of a CMOS compatible bistable electromagnetic microvalve with latching/unlatching mechanism on a single wafer," J. Micromech. Microeng., vol. 16, no. 2, pp. 266-275, Jan. 2006.

[6] B. Bae, J. Han, R. I. Masel, and M. A. Shannon, "A bidirectional electrostatic microvalve with microsecond switching performance," J. Microelectromech. Syst., vol. 16, no. 6, pp. 1461-1471, Dec. 2007.

[7] I. Fazal and M. C. Elwenspoek, "Design and analysis of a high pressure piezoelectric actuated microvalve," J. Micromech. Microeng., vol. 17, no. 11 , pp. 2366-2379, Oct. 2007.

[8] P.-J. Chen, D. C. Rodger, E. Meng, M. S. Humayun, and Y.-C. Tai, "Surface-micromachined parylene dual valves for on-chip unpowered microflow regulation," J. Microelectromech. Syst., vol. 16, no. 2, pp. 223231, Apr. 2007.

[9] P.-J. Chen and Y.-C. Tai, "Floating-disk parylene micro check valve," in Proc. 20th IEEE Int. Conf. MEMS, Kobe, Japan, Jan. 21-25, 2007, pp. 453-456.

[10] P.-J. Chen, D. C. Rodger, M. S. Humayun, and Y.-C. Tai, "Floatingdisk parylene microvalve for self-regulating biomedical flow controls," in Proc. 21st IEEE Int. Conf. MEMS, Tucson, AZ, Jan. 13-17, 2008, pp. $575-578$.

[11] P. Cousseau, R. Hirschi, B. Frehner, S. Gamper, and D. Maillefer, "Improved micro-flow regulator for drug delivery systems," in Proc. 14th IEEE Int. Conf. MEMS, Interlaken, Switzerland, Jan. 21-25, 2001, pp. 527-530.

[12] R. S. Ayyala, J. L. Duarte, and N. Sahiner, "Glaucoma drainage devices: State of the art," Expert Rev. Med. Devices, vol. 3, no. 4, pp. 509-521, Jul. 2006

[13] B. Bae, K. Park, and M. A. Shannon, "MEMS application of actuators and sensors for glaucoma treatment," in MEMS/NEMS Handbook: Techniques and Applications, vol. 5, C. T. Leondes, Ed. New York: Springer-Verlag, 2006, pp. 57-99.

[14] T. Pan, M. S. Stay, V. H. Barocas, J. D. Brown, and B. Ziaie, "Modeling and characterization of a valved glaucoma drainage device with implications for enhanced therapeutic efficacy," IEEE Trans. Biomed. Eng., vol. 52, no. 5, pp. 948-951, May 2005.

[15] P. V. Loeppert and S. B. Lee, "SiSonic ${ }^{\mathrm{TM}}$-The First Commercialized MEMS microphone," in Proc. 12th Solid-State Sens., Actuators, Microsyst. Workshop, Hilton Head Island, SC, Jun. 4-8, 2006, pp. 27-30.

[16] Certificate of Compliance, USP Biological Tests, North Amer. Sci. Assoc., Inc., Northwood, OH, 2005.

[17] L. Wolgemuth, "Assessing the performance and suitability of parylene coating," Med. Device Diagn. Ind., vol. 22, pp. 42-49, Aug. 2000. 
[18] T. A. Harder, T.-J. Yao, Q. He, C.-Y. Shih, and Y.-C. Tai, "Residual stress in thin-film parylene C," in Proc. 15th IEEE Int. Conf. MEMS, Las Vegas, NV, Jan. 20-24, 2002, pp. 435-438.

[19] S. Timoshenko and S. Woinowsky-Krieger, Theory of Plates and Shells, 2nd ed. New York: McGraw-Hill, 1959.

[20] W. C. Young, ROARK's Formulas for Stress \& Strain, 6th ed. New York: McGraw-Hill, 1989.

[21] P.-J. Chen, D. C. Rodger, S. Saati, J. C. Altamirano, C.-H. Lin, R. Agrawal, R. Varma, M. S. Humayun, and Y.-C. Tai, "Implementation of microfabricated suture-less flexible parylene tissue anchors on minimally invasive biomedical implants," in Proc. 11th Int. MicroTAS Conf., Paris, France, Oct. 7-11, 2007, pp. 518-520.

[22] J. C. Lin, P.-J. Chen, S. Saati, R. Varma, M. Humayan, and Y.-C. Tai, "Implantable microvalve-packaged glaucoma drainage tube," in Proc. 13th Solid-State Sens., Actuators, Microsyst. Workshop, Hilton Head Island, SC, Jun. 1-5, 2008, pp. 146-149.

[23] X. Q. Wang, Q. Lin, and Y.-C. Tai, "A parylene micro check valve," in Proc. 12th IEEE Int. Conf. MEMS, Orlando, FL, Jan. 17-21, 1999, pp. $177-182$.

[24] J. Xie, X. Yang, X. Q. Wang, and Y.-C. Tai, "Surface micromachined leakage proof parylene check valve," in Proc. 14th IEEE Int. Conf. MEMS, Interlaken, Switzerland, Jan. 21-25, 2001, pp. 539-542.

[25] T.-J. Yao, X. Yang, and Y.-C. Tai, "BrF 3 dry release technology for large freestanding parylene microstructures and electrostatic actuators," Sens. Actuators A, Phys., vol. 97/98, pp. 771-775, Apr. 2002.

[26] J. Xie, J. Shih, Q. Lin, B. Yang, and Y.-C. Tai, "Surface micromachined electrostatically actuated micro peristaltic pump," Lab Chip, vol. 4, no. 5, pp. 495-501, Oct. 2004

[27] J. Shih, J. Xie, and Y.-C. Tai, "Surface micromachined and integrated capacitive sensors for microfluidic applications," in Proc. 12th Int. Transducers Conf., Boston, MA, Jun. 8-12, 2003, pp. 388-391.

[28] T. Gervais, J. El-Ali, A. Günther, and K. F. Jensen, "Flow-induced deformation of shallow microfluidic channels," Lab Chip, vol. 6, no. 4, pp. 500507, Apr. 2006.

[29] Q. He, C. Pang, Y.-C. Tai, and T. D. Lee, "Ion liquid chromatography ona-chip with beads-packed parylene column," in Proc. 17th IEEE Int. Conf. MEMS, Maastricht, The Netherlands, Jan. 25-29, 2004, pp. 212-215.

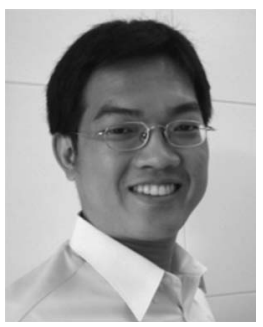

Po-Jui Chen (S'03) was born in Taipei, Taiwan, in 1980 . He received the B.S. degree in mechanical engineering from National Taiwan University, Taipei, in 2002, and the M.S. degree in electrical engineering from the California Institute of Technology (Caltech), Pasadena, in 2004, where he is currently working toward the Ph.D. degree in electrical engineering in the area of microelectromechanical systems (MEMS) in the Department of Electrical Engineering, Division of Engineering and Applied Science.

He has been with Dr. Yu-Chong Tai's Caltech Micromachining Laboratory as a Graduate Researcher since 2003. He has published more than 20 peerreviewed journal and conference papers and is the holder of several U.S. patent applications. His research interests include implantable biomedical microdevices, integrated microfluidic and laboratory-on-a-chip systems, MEMS sensors/actuators/structures, and micro-/nanofabrication technologies.

$\mathrm{Mr}$. Chen is a student member of the American Society of Mechanical Engineers and a full member of Sigma Xi. He was elected as an honorary member of Phi Tau Phi in Taiwan in 2002. He is the recipient of several scholarships, the Sorenson Fellowship, the Outstanding College Youth Award at National Taiwan University in 2002, and the CHEMINAS Best Student Poster Award in the International MicroTAS 2006 Conference in Tokyo, Japan.

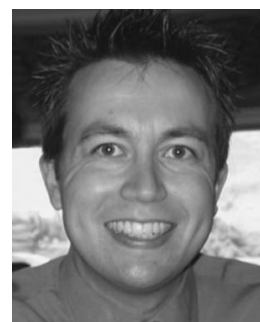

Damien C. Rodger (S'97-M'08) received the B.S. degree in electrical engineering (magna cum laude with honors) from Cornell University, Ithaca, NY, in 2000 , and the $\mathrm{Ph} . \mathrm{D}$. degree in bioengineering from the California Institute of Technology, Pasadena, in 2008. He is currently working toward the M.D. degree in the Keck School of Medicine, University of Southern California, Los Angeles, while conducting research on microelectrode technologies for retinal and spinal cord prostheses and on other novel bioMEMS for ophthalmic use.

Dr. Rodger held a Whitaker Foundation Graduate Fellowship from 2003 to 2006 and is a member of the Association for Research in Vision and Ophthalmology.

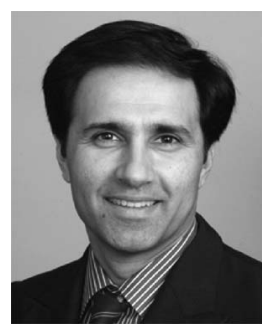

Mark S. Humayun (M'97) received the B.S. degree from Georgetown University, Washington, DC, in 1984, the M.D. degree from Duke University, Durham, NC, in 1989, and the Ph.D. degree from the University of North Carolina, Chapel Hill, in 1994. He finished his training by completing an Ophthalmology Residency at Duke University and a Fellowship in Vitreoretinal Diseases at Johns Hopkins Hospital.

Currently, he is a Professor of Ophthalmology, Biomedical Engineering, and Cell and Neurobiology with the Keck School of Medicine, University of Southern California, Los Angeles, where he is also the Director of the National Science Foundation Biomimetic Microelectronic Systems Engineering Research Center. He is also with the Doheny Eye Institute, Los Angeles. He is also the Director of the Department of Energy Artificial Retina Project that is a consortium of five Department of Energy laboratories and four universities, as well as industry.

Dr. Humayun is a member of the Association for Research in Vision and Ophthalmology, Biomedical Engineering Society, Vitreous Society, and Retina Society, and was elected as a Fellow of the American Institute for Medical and Biological Engineering in 2007.

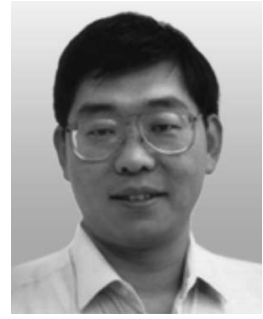

Yu-Chong Tai (M'97-SM'03-F'06) received the B.S. degree in electrical engineering from National Taiwan University, Taipei, Taiwan, in 1981, and the M.S. and Ph.D. degrees in electrical engineering from the University of California, Berkeley, in 1986 and 1989, respectively.

$\mathrm{He}$ is with the Department of Electrical Engineering, Division of Engineering and Applied Science, California Institute of Technology (Caltech), Pasadena, where he founded the Caltech Micromachining Laboratory and currently is a Professor of Electrical Engineering, Mechanical Engineering, and Bioengineering. He is also with the Department of Bioengineering, Division of Engineering and Applied Science, Caltech. He has published more than 300 technical articles in the microelectromechanical systems (MEMS) field. His current research interests include flexible MEMS, bioMEMS, MEMS for retinal implants, parylenebased integrated microfluidics, neuroprobes/neurochips, and high performance liquid chromatography-based laboratory-on-a-chip systems.

Dr. Tai is the recipient of an IBM Fellowship, the David and Lucile Packard Fellowship, Ross Tucker Award, David Sakrison (Best Thesis Award), Presidential Young Investigator Award, Packard Award, Association for Laboratory Automation Achievement Award, Caltech Teaching Award, and several Prize Paper Awards. He cochaired the IEEE International MEMS 2002 Conference in Las Vegas, NV. He is a Fellow of the Institute of Physics. 